\section{Bemerkungen über belastete Integralgleichungen}

von

\section{LEON LICHTENSTEIN (in Leipzig).}

Manche Probleme der mathematischen Physik führen auf Integralgleichungen, bei denen die Integration sich nicht nur über das eigentliche der Betrachtung zugrunde liegende Gebiet, sondern darüber hinaus auch noch über seine Berandung oder sonstige ausgezeichnete Flächen oder Linien erstreckt. Handelt es sich etwa um ein zweidimensionales Gebiet, so treten auf der linken Seite der Gleichung neben Doppelintegralen auch noch einfache Integrale auf.

Es sei etwa $T$ ein von einer stetig gekrümmten Kurve $S$ begrenztes Gebiet, und es möge $S_{0}$ einen stetig gekrümmten Querschnitt bezeichnen, der $T$ in die beiden Teilgebiete $T_{1}$ und $T_{2}$ zerlegt. Es seien weiter $a$ und $b$ zwei sowohl im $T_{1}$ und auf seinem Rande, als auch in $T_{2}$ mit Einschluß des Randes nebst ihren partiellen Ableitungen erster Ordnung stetige Funktionen, die auf $S_{0}$ entsprechend $a_{1}, b_{1}$ und $a_{2}, b_{2}$ zu Randwerten haben, $\left(a_{2}-a_{1}\right)^{2}+\left(b_{2}-b_{1}\right)^{2} \neq 0$. Es seien schließlich $c$ und $f$ beliebige in $T+S$ stetige, in $T$ der HöLderschen Bedingung genügende Funktionen. Betrachten wir die partielle Differentialgleichung

$$
\frac{\partial^{2} u}{\partial x^{2}}+\frac{\partial^{2} u}{\partial y^{2}}+a \frac{\partial u}{\partial x}+b \frac{\partial u}{\partial y}+c u=f
$$

und suchen diejenige in $T$ nebst ihren partiellen Ableitunger erster Ordnung stetige Lösung $u(x, y)$ zu bestimmen, die in 7 und $T_{2}$ stetige partielle Ableitungen zweiter Ordnung hat un auf $S$ verschwindet.

Es sei $G(\xi, \eta ; x, y)$ die zu $T$ gehörige, auf $S$ verschwin dende GREENsche Funktion der Potentialtheorie. Nach bekannten
Sätzen folgt aus (1) für alle $(\xi, \eta)$ in $T$

(2) $u(\xi, \eta)=\frac{1}{2 \pi} \int_{T} G(\xi, \eta ; x, y)\left[-f(x, y)+a(x, y) \frac{\partial u}{\partial x}+b(x, y) \frac{\partial: z}{\partial y}\right.$

$$
+c(x, y) u(x, y)] d x d y \text {, }
$$

und nach einer teilweisen Integration

$$
\text { (3) } \begin{aligned}
u(\xi, \eta) & =-\frac{1}{2 \pi} \int_{T}\left\{\frac{\partial}{\partial x}[a(x, y) G(\xi, \eta ; x, y)]+\frac{\partial}{\partial y}\left[b(x, y) G\left(\xi, \eta_{i} ; x, y\right)\right]\right. \\
- & c(x, y)\} u(x, y) d x d y-\frac{1}{2 \pi} \int_{S_{0}}\left[\left(a_{2}-a_{1}\right) \frac{d y}{d s}-\left(b_{2}-b_{1}\right) \frac{d x}{d s}\right] \\
& \times G(\xi, \eta ; x, y) u(x, y) d s-\frac{1}{2 \pi} \int_{7} G(\xi, \eta ; x, y) f(x, y) d x d y .
\end{aligned}
$$

Bei der Integration längs $S_{0}$ bleibt hierbei $T_{1}$ linker Hand liegen ${ }^{\mathrm{j}}$ ).

Die Beziehung (3) ist eine Integralgleichung von der eingangs genannten Form. Weitere Beispiele bietet die Theorie der Gleichgewichtsfiguren rotierender nichthomogener Flüssigkeiten, insbesondere die Theorie der Gestalt der Erde ${ }^{2}$ ).

Es sei, wie vorhin, $T$ ein von einer stetig gekrümmten Kurve $S$ begrenztes endliches Gebiet in der Ebene der Variablen $x, y$. Es sei $\stackrel{1}{K}\left(\tau, \tau^{\prime}\right)$ eine für alle $\tau$ und $\tau^{\prime}$ in $T+S$ erklärte stetige Funktion, und es möge $\stackrel{2}{K}\left(\tau, \sigma^{\prime}\right)$ eine für alle $\tau$ in $T+S$ und alle $\sigma^{\prime}$ auf $S$ erklärte stetige Funktion, $f(\tau)$ eine beliebige in $T+S$ stetige Funktion bezeichnen. Die Integralgleichung

(4) $\varphi(\tau)+\lambda \int_{T} \frac{1}{K}\left(\tau, \tau^{\prime}\right) \varphi\left(\tau^{\prime}\right) d \tau^{\prime}+\lambda \int_{S}^{2} K\left(\tau, \sigma^{\prime}\right) \varphi\left(\sigma^{\prime}\right) d \sigma^{\prime}=f(\tau)$,

eine "belastete Integralgleichung" in der Bezeichnungsweise von A. $\mathrm{KNESER}^{3}$ ), läßt sich, wenn die Integralgleichung

1) Vgl. L. Lichtenstein, Randwertaufgaben der Theorie der linearen partiellen Differentialgleichungen zweiter Ordnung vom elliptischen Typus, II, Journal für Mathematik 143 (1913) p. 51-105, insbesondere p. 55.

2) Vgl. meine demnächst erscheinende Arbeit: Untersuchungen über die Gleichrewichtsfiguren rotierender Flüssigkeiten, deren Teilchen einander nach dem Newtonschen Gesetze anziehen. Dritte Abhandlung. Nichthomogene Flüssigkeiten. Figur der. Erde.

3) Vgl. A. Kneser, Belastete Integralgleichungen, Rendiconti del Circolo Matematico di Palermo 37 (1914) p. 169-197. Dort wird den Betrachtungen der eindimensionale Fall zugrunde gelegt und dementsprechend Gleichungen von 


$$
\varphi(\tau)+\lambda \int_{T}^{1} K\left(\tau, \tau^{\prime}\right) \varphi\left(x^{\prime}\right) d x^{\prime}=0
$$

\section{$\S 1$.}

keine Nullösung hat, wie man leicht sieht, ohne weiteres auf eine gewöhnliche Integralgleichung zurückführen. Ist nämlich $\stackrel{1}{L}\left(\tau, \tau^{\prime}\right)$ der zu $\lambda \stackrel{1}{K}\left(\tau, \tau^{\prime}\right)$ gehörige lösende Kern, so folgt aus (4)

$$
\text { (6) } \begin{gathered}
\varphi(\tau)=f(\tau)-\lambda \int_{S}^{2} K\left(\tau, \sigma^{\prime}\right) \varphi\left(\sigma^{\prime}\right) d \sigma^{\prime}-\int_{T}^{1} L\left(\tau, \tau^{\prime}\right) f\left(\tau^{\prime}\right) d \tau^{\prime} \\
+\lambda \int_{T} \int_{S}^{1} L\left(\tau, \tau^{\prime}\right) \stackrel{2}{K}\left(\tau^{\prime}, \sigma^{\prime}\right) \varphi\left(\sigma^{\prime}\right) d \tau^{\prime} d \sigma^{\prime}
\end{gathered}
$$

mithin für $\tau \rightarrow \sigma$ auf $S$

$$
\begin{gathered}
(7) \quad \varphi(\sigma)=f(\sigma)-\lambda \int_{S} K_{S}^{2}\left(\sigma, \sigma^{\prime}\right) \varphi\left(\sigma^{\prime}\right) d \sigma^{\prime}-\int_{T}^{1} L\left(\sigma, \tau^{\prime}\right) f\left(\tau^{\prime}\right) d \tau^{\prime} \\
+\lambda \int_{T} \int_{S}^{1} L\left(\sigma, \tau^{\prime}\right) \stackrel{2}{K}\left(\tau^{\prime}, \sigma^{\prime}\right) \varphi\left(\sigma^{\prime}\right) d \tau^{\prime} d \sigma^{\prime}
\end{gathered}
$$

und dies ist eine FREDHOLMsche Integralgleichung im üblichen Sinne.

Ist aus (7) $\varphi(\sigma)$ ermittelt, so liefert (6) $\varphi(\tau)$ für alle $\tau$ in $T+S$. Es ist leicht zu sehen, daß, wie verlangt werden muß, auf $S$ die beiden Bestimmungen der gesuchten Lösung denselben Wert ergeben.

Nach einer Bemerkung von A. KNESER ${ }^{4}$ ) gelten bei einer belasteten Integralgleichung bei geeigneter Schreibweise die klassischen FREDHoLmschen Auflösungsformeln. Wir werden dies im Folgenden an Hand der Integralgleichung (4) zeigen. Unsere Ausführungen, deren Ergebnisse kaum etwas wesentlich neues enthalten, sind hauptsächlich darum zu Papier gebracht worden, um für die eingangs erwähnten Anwendungen auf die Theorie der Gleichgewichtsfiguren rotierender Flüssigkeiten eine sichere Grundlage zu schaffen.

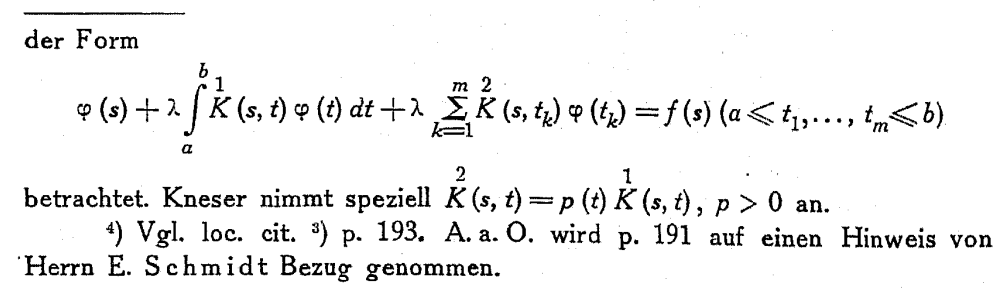

Es sei $h>0$ eine hinreichend kleine Zahl, und es möge $S_{h}$ die zu $S$ im Abstande $h$ parallele Kurve in $T$ bezeichnen. Das von $S_{h}$ begrenzte, ganz im Innern von $T$ gelegene Gebiet heiße $T_{h}$. Wir setzen

$$
K_{h}\left(\tau, \tau^{\prime}\right)=\left\{\begin{array}{l}
\frac{1}{K}\left(\tau, \tau^{\prime}\right) \text { in } T_{h}, \\
\frac{1}{K}\left(\tau, \tau^{\prime}\right)+\frac{1}{h} \stackrel{2}{K}\left(\tau, \sigma^{\prime}\right) \text { in } T-T_{h},
\end{array}\right.
$$

unter $\sigma^{\prime}$ der Fußpunkt des von $\tau^{\prime}$ auf $S$ gefällten Lotes verstanden, und betrachten die Integralgleichung

$$
\varphi_{h}(\tau)+\lambda \int_{T} K_{h}\left(\tau, \tau^{\prime}\right) \varphi_{h}\left(\tau^{\prime}\right) d \tau^{\prime}=f(\tau) .
$$

Augenscheinlich gilt, wenn $\chi(\tau)$ irgendeine in $T+S$ erklärte stetige Funktion bezeichnet,

$$
\begin{aligned}
\lim _{h \rightarrow 0} \int_{T} K_{h}\left(\tau, \tau^{\prime}\right) \chi\left(\tau^{\prime}\right) d \tau^{\prime}=\int_{T}^{1} K\left(\tau, \tau^{\prime}\right) \chi\left(\tau^{\prime}\right) d \tau^{\prime} \\
+\int_{S}^{2} K\left(\tau, \sigma^{\prime}\right) \chi\left(\sigma^{\prime}\right) d \sigma^{\prime},
\end{aligned}
$$

und zwar für alle $\tau$ in $T+S$ gleichmäßig. Es ist darum zu vermuten, daß die Löșung der Integralgleichung (9) für $h \rightarrow 0$ diejenige der belasteten Integralgleichung (4) liefern dürfte.

Es sei $H_{h}\left(\tau, \tau^{\prime} ; \lambda\right)$ der zu dem Kerne $K_{h}\left(\tau, \tau^{\prime}\right)$ gehörige FREDHOLMSche Quotient

$$
H_{h}\left(\tau, \tau^{\prime} ; \lambda\right)=\frac{D_{h}\left(\tau, \tau^{\prime} ; \lambda\right)}{D_{h}(\lambda)}=\frac{\sum_{m=0}^{\infty} \frac{\lambda^{m}}{m !} A_{m}^{(h)}\left(\tau, \tau^{\prime}\right)}{1+\sum_{m=1}^{\infty} \frac{\lambda^{m}}{m !} A_{m-1}^{(h)}},
$$

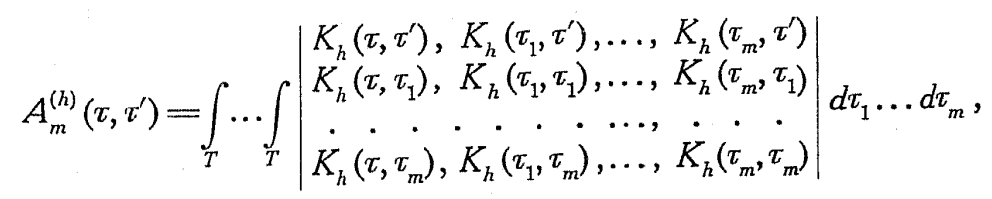

$$
A_{m}^{(h)}=\int_{1} A_{m}^{(h)}\left(\tau_{0}, \tau_{0}\right) d \tau_{0}
$$


Nach (8) kann $K_{h}\left(\tau, \tau^{\prime}\right)$ als Summe der beiden Kerne

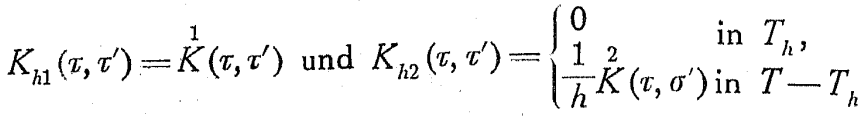

aufgefaßt werden ${ }^{5}$ ). Wir können darum auch schreiben

$$
A_{m}^{(h)}\left(\tau, \tau^{\prime}\right)=\stackrel{1}{A}_{m}^{(h)}\left(\tau, \tau^{\prime}\right)+\frac{1}{h} \stackrel{2}{A_{m}^{(h)}}\left(\tau, \tau^{\prime}\right)
$$

mit

(15)

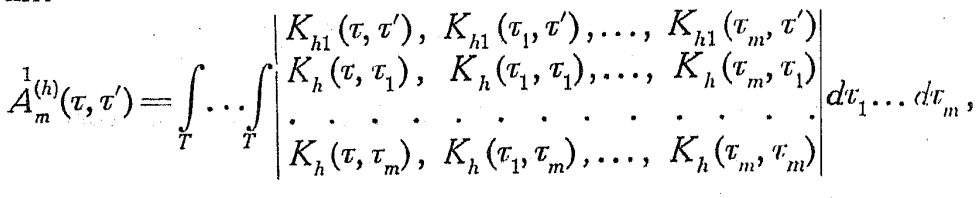

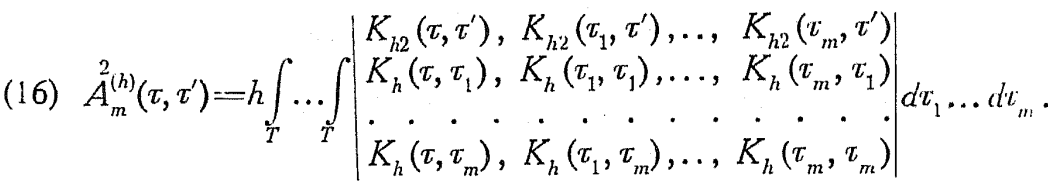

Die $m$-fachen Integrale (15) und (16) zerfallen in $2^{m}$ Summanden, indem in einer Anzahl Zeilen $K_{h}$ durch $K_{h 1}$, in dem Rest durch $K_{h 2}$ ersetzt wird. Es sei

$$
\operatorname{Max}\left\{\left|\stackrel{1}{K}\left(\tau, \tau^{\prime}\right)\right|,\left|\stackrel{2}{K}\left(\tau, \sigma^{\prime}\right)\right|\right\}=M .
$$

Betrachten wir denjenigen der $2^{m}$ Bestandteile etwa von $\stackrel{1}{(m)}_{m}^{(h)}\left(\tau, \tau^{\prime}\right)$, in dem $K_{h 1}$, sagen wir, $m_{1}$-mal, $K_{h 2}$ dagegen $\dot{m}_{2}=m-m_{1}$-mal vertreten ist. Dem Hadamardschen Determinantensatze gemäß ist der absolute Betrag der zu integrierenden Funktion

$$
\left.\leqslant m^{\frac{m}{2}} M^{m} \frac{1}{h^{m_{3}}}{ }^{0}\right) \text {. }
$$

Der betrachtete Term ist $m_{1}$-mal über $T, m_{2}$-mal über $T-T_{h}$ zu integrieren. Der Inhalt des $m$-dimensionalen Gebietes hat den Wert

$$
\Theta^{m_{1}} A^{m_{2}} h^{m_{2}}(1+\delta(h))^{m_{2}},
$$

5) Auch jetzt bezeichnet $\sigma^{\prime}$ den Fußpunkt des von $\tau^{\prime}$ auf $S$ gefällten Lotes. 6) Man beachte, daß in $m_{2}$ Spalten $1 / \mathrm{h}$ als gemeinsamer Faktor auftritt. Nach Ausklammern dieses Faktors erhält man für den Betrag der übrig bleibenden Determinante die Schranke $m^{\frac{m}{2}} M^{m}$. unter $\Theta$ den Flächeninhalt von $T$, unter $A$ die Länge von $S$, unter $\delta(h)$ eine mit $h$ zugleich verschwindende Größe verstanden. Das Ergebnis der Integration ist also absolut

$$
\begin{gathered}
\leqslant m^{\frac{m}{2}} M^{m^{m}} \frac{1}{h^{m_{2}}} \Theta^{m_{1}} \mathcal{L}^{m_{2}} h^{m_{2}}(1+\delta(h))^{m_{2}} \\
\leqslant m^{\frac{m}{2}} M^{m} \Theta^{m_{1}} \mathcal{L}^{m_{2}}(1+\delta(h))^{m_{2}} .
\end{gathered}
$$

Es sei $h_{0}$ so klein gewählt, daß für $h \leqslant h_{0}$ gewiß $\mid \delta(h)<1$ wird. Wird zur Vereinfachung

$$
\operatorname{Max}\{\Theta, A\}=\Theta_{*}
$$

gesetzt, so kann die Schranke (18) durch

$$
2^{m} m^{\frac{m}{2}} M^{m} \Theta_{*}^{m}
$$

ersetzt werden. Da $A_{m}^{(h)}\left(\tau, \tau^{\prime}\right)$ in $2^{m}$ Summanden der soeben betrachteten Art zerfällt, so finden wir, alles in allem,

$$
\left|\stackrel{1}{A}_{m}^{(h)}\left(\tau, \tau^{\prime}\right)\right| \leqslant 2^{2 m} m^{\frac{m}{2}} M^{m} \Theta_{*}^{m} .
$$

Ebenso ist

$$
\left|\stackrel{2}{A}_{m}^{(h)}\left(\tau, \tau^{\prime}\right)\right| \leqslant 2^{2 m} m^{\frac{m}{2}} M^{m} \Theta_{*}^{m} .
$$

Der Zerlegung (14) entsprechend läßt sich $D_{h}\left(\tau, \tau^{\prime} ; \hat{t}\right)$ in der Form

$$
D_{h}\left(\tau, \tau^{\prime} ; \lambda\right)=L_{h}^{1}(\tau, \tau ; \lambda)+\frac{1}{h} \stackrel{2}{D}_{h}\left(\tau, \tau^{\prime} ; \lambda\right)
$$

darstellen. Augenscheinlich konvergieren die beiden Reihen $\stackrel{1}{D}\left(\tau, \tau^{\prime} ; \lambda\right)$ und $\stackrel{2}{\nu}\left(\tau, \tau^{\prime} ; \lambda\right)$ für alle $h \leqslant h_{\mathrm{i}}$ und alle $\lambda$ gleichmäßig. Für $h \rightarrow 0$ geht, wie man leicht sieht, $\stackrel{1}{D}_{h}\left(\tau, \tau^{\prime} ; \lambda\right)$ für alle reellen oder komplexen $\lambda$ mit $|\lambda| \leqslant \lambda_{0}\left(\lambda_{1}\right.$ beliebig $)$ gleichmäßig gegen den Ausdruck

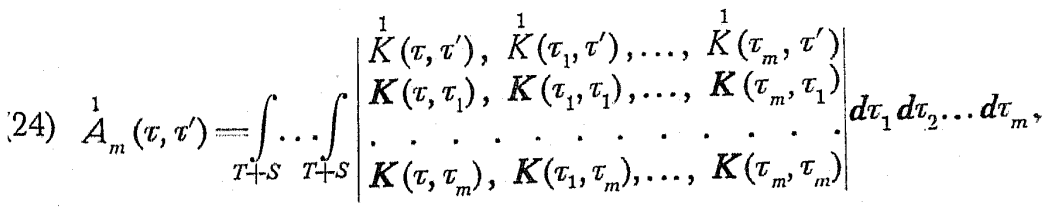


in $\operatorname{dem} K\left(\tau, \tau_{1}\right)$ und $d \tau$ folgende Bedeutung haben. Es ist

$$
\begin{aligned}
K\left(\tau, \tau_{1}\right)= & \left\{\begin{array}{l}
\dot{K}\left(\tau, \tau_{1}\right) \text { für } \tau_{1} \text { in } T, \\
2 \\
K\left(\tau, \sigma_{2}\right) \text { für } \tau_{1}=\sigma_{1} \text { auf } S,
\end{array}(\tau \text { in } T+S)\right. \\
& \boldsymbol{d} \tau=d \tau \text { in } T,=d \sigma \text { auf } S .
\end{aligned}
$$

Der Integralausdruck $\stackrel{\prime}{A}_{m}\left(\tau, \tau^{\prime}\right)$ zerfällt in $2^{m}$ Summanden der vorhin betrachteten Zerfällung des Ausdruckes $A_{m}^{(h)}$ entsprechend. Die Integration erstreckt sich bald über $T$, bald über $S$. So ist beispielweise

$$
\begin{aligned}
& \stackrel{1}{A}_{0}\left(\tau, \tau^{\prime}\right)=\stackrel{1}{K}\left(\tau, \tau^{\prime}\right), \\
& \stackrel{1}{A_{1}}\left(\tau, \tau^{\prime}\right)=\int_{T+S}\left|\begin{array}{l}
\stackrel{1}{K}\left(\tau, \tau^{\prime}\right), \stackrel{1}{K}\left(\tau_{1}, \tau^{\prime}\right) \\
K\left(\tau, \tau_{1}\right), K\left(\tau_{1}, \tau_{1}\right)
\end{array}\right| d \tau_{1}
\end{aligned}
$$

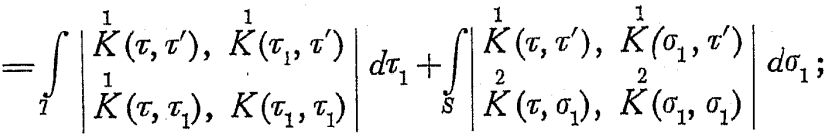

$\stackrel{1}{D}\left(\tau, \tau^{\prime} ; \lambda\right)$ ist eine ganze transzendente Funktion von $\lambda$.

Ganz analog geht $\stackrel{2}{D}\left(\tau, \sigma^{\prime} ; \lambda\right)$ für $h \rightarrow 0$, wie vorhin, für alle $\lambda$ mit $|\lambda| \leqslant \lambda_{0}$ gleichmäßig gegen die ganze transzendente Funktion

$$
\stackrel{2}{D}\left(\tau, \sigma^{\prime} ; \lambda\right)=\sum_{m=0}^{\infty} \frac{\lambda^{m}}{m !} \stackrel{2}{A}\left(\tau, \sigma^{\prime}\right)
$$

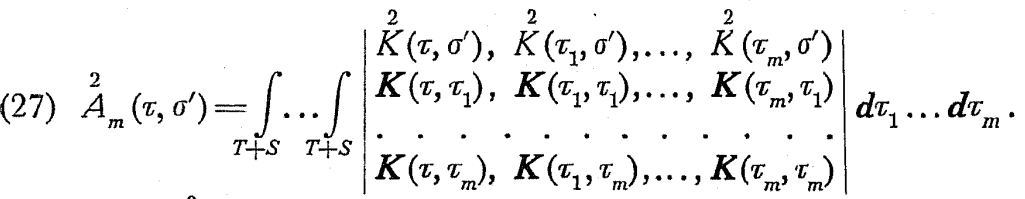

Auch $\left.\stackrel{2}{A}_{m}\left(\tau, \sigma^{\prime}\right)^{7}\right)$ zerfällt in $2^{m}$ Summandem, und es ist beispielweise

$$
\begin{aligned}
& A_{0}\left(\tau, \sigma^{\prime}\right)=\stackrel{2}{K}\left(\tau, \sigma^{\prime}\right),
\end{aligned}
$$

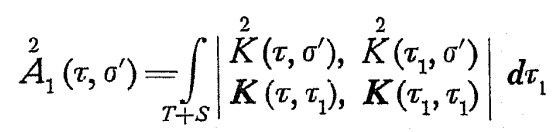

7) Nach (13) ist $\stackrel{2}{A}_{m}\left(\tau, \tau^{\prime}\right)$ nur für $\tau^{\prime}=\sigma^{\prime}$ von Null verschieden.

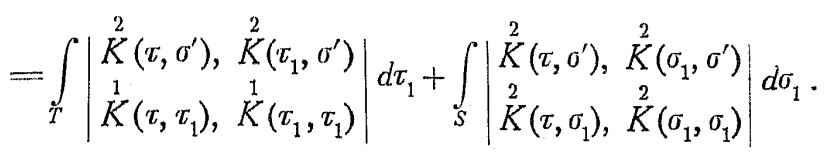

Wir setzen jetzt zur Abkürzung

$$
\begin{aligned}
A_{m}\left(\tau, \tau_{1}\right)= & \left\{\begin{array}{l}
\stackrel{1}{A}_{m}\left(\tau, \tau_{1}\right) \text { für } \tau_{1} \text { in } T, \\
\stackrel{2}{A}_{m}\left(\tau, \sigma_{1}\right) \text { für } \tau_{1}=\sigma_{1} \text { auf } S,
\end{array}(\tau \text { in } T+S)\right. \\
& \boldsymbol{D}\left(\tau, \tau^{\prime} ; \lambda\right)=\sum_{m=0}^{\infty} \frac{\lambda^{m}}{m !} A_{m}\left(\tau, \tau^{\prime}\right),
\end{aligned}
$$

kürzer

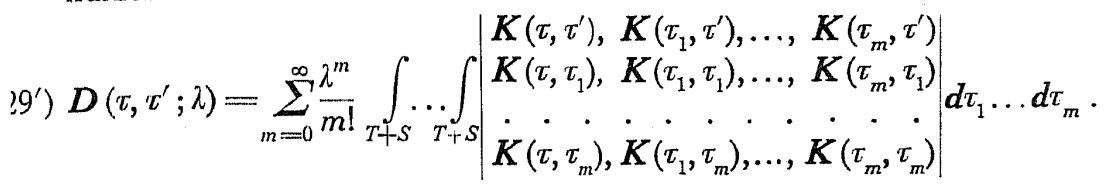

Es gilt augenscheinlich

$$
\begin{aligned}
& \boldsymbol{D}\left(\tau, \tau^{\prime} ; \lambda\right)=\stackrel{1}{D}\left(\tau, \tau^{\prime} ; \lambda\right) \text { für alle } \tau^{\prime} \text { in } T, \\
& \left.\boldsymbol{D}\left(\tau, \sigma^{\prime} ; \lambda\right)=\stackrel{2}{D}\left(\tau, \sigma^{\prime} ; \lambda\right)^{\mathrm{s}}\right) .
\end{aligned}
$$

Man überzeugt sich jetzt ohne Mühe, daß $D_{h}(\lambda)$ für alle $h \leqslant h_{0}$ und alle $\lambda$ mit $|\lambda| \leqslant \lambda_{0}$ für $h \rightarrow 0$ gleichmäßig gegen

$$
\begin{array}{r}
D(\lambda)=1+\sum_{m=1}^{\infty} \frac{\lambda^{m}}{m !} A_{m-1} \quad\left(A_{m}=\int_{T+S} A_{m}\left(\tau_{0}, \tau_{0}\right) d \tau_{0}\right. \\
\left.=\int_{T}^{1} A_{m}\left(\tau_{0}, \tau_{0}\right) d \tau_{0}+\int_{S}^{2} A_{m}\left(\sigma_{0}, \sigma_{0}\right) d \sigma_{0}\right)
\end{array}
$$

konvergiert.

Es sei schlieflich

$$
H\left(\tau, \dot{\tau}^{\prime} ; \lambda\right)=\frac{D\left(\tau, \tau^{\prime} ; \lambda\right)}{D(\lambda)}
$$

Offenbar ist für alle $\tau$ in $T+S$

8) Man vergleiche in diesem Zusammenhang die Ausführungen p. 28-29 in meinen kürzlich erschienenen „Vorlesungen über einige Klassen nichtlinearer Integralgleichungen and Integro-Differentialgleichungen nebst Anwendungen", Berlin 1931. 


$$
\begin{aligned}
& H\left(\tau, \tau^{\prime} ; \lambda\right)=\frac{\stackrel{1}{D}\left(\tau, \tau^{\prime} ; \lambda\right)}{D(\lambda)} \text { für alle } \tau^{\prime} \text { in } T \\
& H\left(\tau, \sigma^{\prime} ; \lambda\right)=\frac{\stackrel{2}{D}\left(\tau, \sigma^{\prime} ; \lambda\right)}{D(\lambda)} .
\end{aligned}
$$

\section{$\S 2$.}

Es möge nun $\lambda$ einen. Wert haben, so daß $D(\lambda) \neq 0$ ist. Dann ist für alle hinreichend kleinen $h$ gewiß auch $D_{h}(\lambda) \neq 0$. Die Integralgleichung (9) hat eine und nur eine Lösung

$$
\varphi_{h}(\tau)=f(\tau)-\lambda \int_{T} H_{h}\left(\tau, \tau^{\prime} ; \lambda\right) f\left(\tau^{\prime}\right) d \tau^{\prime} .
$$

Geht man zur Grenze $h \rightarrow 0$ über, so findet man leicht, daß

$$
\begin{gathered}
\varphi(\tau)=f(\tau)-\lambda \int_{T_{+}^{\prime} S} H\left(\tau, \tau^{\prime} ; \lambda\right) f\left(\tau^{\prime}\right) d \tau^{\prime} \\
=f(\tau)-\lambda \int_{T} \frac{\dot{D}\left(\tau, \tau^{\prime} ; \lambda\right)}{D(\lambda)} f\left(\tau^{\prime}\right) d \tau^{\prime}-\lambda \int_{S}^{2} \frac{D^{2}\left(\tau, \sigma^{\prime} ; \lambda\right)}{D(\lambda)} f\left(\sigma^{\prime}\right) d \sigma^{\prime}
\end{gathered}
$$

eine Lösung der „belasteten“ Integralgleichung (4) darstellt.

Der Übersichtlichkeit halber setzen wir

$$
\Phi(\tau)=\varphi(\tau), \quad F(\tau)=f(\tau) .
$$

Wegen (25) können wir für (4) auch kürzer schreiben

$$
\Phi(\tau)+\lambda \int_{T+S} K\left(\tau, \tau^{\prime}\right) \Phi\left(\tau^{\prime}\right) d \tau^{\prime}=F(\tau) .
$$

Für (35) tritt dementsprechend

$$
\Phi(\tau)=F(\tau)-\lambda \int_{T+S} H\left(\tau, \tau^{\prime} ; \lambda\right) F\left(\tau^{\prime}\right) d \tau^{\prime}
$$

ein.

Die zu (9) adjungierte Integralgleichung

$$
\psi_{h}\left(\tau^{\prime}\right)+\lambda \int_{T} K_{h}\left(\tau, \tau^{\prime}\right) \psi_{h}(\tau) d \tau=f\left(\tau^{\prime}\right)
$$

hat, wenn wir wie vorhin $D(\lambda) \neq 0$ voraussetzen,

$$
\psi_{h}\left(\tau^{\prime}\right)=f\left(\tau^{\prime}\right)-\lambda \int_{i} H_{h}\left(\tau, \tau^{\prime} ; \lambda\right) f(\tau) d \tau
$$

zur Lösung. Wir wollen jetzt sehen, vias diese Formeln für $h \rightarrow 0$ ergeben.

Wir bemerken vor allem, daß man $f(\tau)$ abteilungsweise stetig annehmen darf, in welchem Falle natürlich auch $\psi_{h}(\tau)$ abteilungsweise stetig ausfallen wird, ersetzen $f(\tau)$ in $T_{h}$ durch $h f_{h}^{*}$ und schreiben

$$
\psi_{h}(\tau)=h \psi_{h}^{*} \text { in } T_{h} .
$$

Die Gleichung (37) liefert dann für $\tau^{\prime}$ in $T_{h}$

(40) $h \psi_{h}^{*}\left(\tau^{\prime}\right)+h \lambda \int_{T_{h}} K_{h}^{1}\left(\tau, \tau^{\prime}\right) \psi_{h}^{*}(\tau) d \tau+\lambda \int_{T-T_{h}} K_{h}^{1}\left(\tau, \tau^{\prime}\right) \psi_{h}(\tau) d \tau=h f^{*}\left(\tau^{\prime}\right)$, für $\tau^{\prime}$ in $T-T_{h}$ hingegen

$$
\begin{gathered}
\psi_{h}\left(\tau^{\prime}\right)+h \lambda \int_{T_{h}}^{1} K\left(\tau, \tau^{\prime}\right) \psi_{h}^{*} d \tau+\lambda \int_{T-T_{h}}^{1} K\left(\tau, \tau^{\prime}\right) \psi_{h}(\tau) d \tau \\
+\lambda \int_{T_{h}} \stackrel{2}{K}_{h}\left(\tau ; \tau^{\prime}\right) \psi_{h}^{*}(\tau) d \tau+\frac{1}{h} \int_{T-T_{h}}^{2} \stackrel{2}{K}_{h}\left(\tau, \tau^{\prime}\right) \psi_{h}(\tau) d \tau=f(\tau) .
\end{gathered}
$$

Wie sich alsbald zeigen wird, konvergiert $\psi_{h}^{*}\left(\tau^{\prime}\right)$ für $h \rightarrow 0$ für alle $\tau^{\prime}$ in jedem ganz in $T$ enthaltenen Bereiche gleichmäBig gegen eine in $T+S$ stetige Funktion $\psi^{*}\left(\tau^{\prime}\right)$. Des weiteren geht $\psi_{h}\left(\tau^{\prime}\right)$ in $T-T_{h}$ gleichmäßig gegen eine auf $S$ erklärte stetige Funktion $\psi\left(\sigma^{\prime}\right)$. Aus (40) und (41) ergibt sich, wie man leicht sieht,

$\left(40^{\prime}\right) \quad \psi^{*}\left(\tau^{\prime}\right)+\lambda \int_{T}^{1} K\left(\tau, \tau^{\prime}\right) \psi^{*}(\tau) d \tau+\lambda \int_{S}^{1} K\left(\sigma, \tau^{\prime}\right) \psi(\sigma) d \sigma=f^{*}\left(\tau^{\prime}\right)$,

$\left(41^{\prime}\right) \quad \psi\left(\sigma^{\prime}\right)+\lambda \int_{T}^{2} K\left(\tau, \sigma^{\prime}\right) \psi^{*}(\tau) d \tau+\lambda \int_{S}^{2} K\left(\sigma, \sigma^{\prime}\right) \psi(\sigma) d \sigma=f\left(\sigma^{\prime}\right)$.

Wir haben auf p. 218 die sprungweise unstetige Funktion

$$
K\left(\tau, \tau_{1}\right)=\left\{\begin{array}{l}
1 \\
K\left(\tau, \tau_{1}\right) \text { für } \tau_{1} \text { in } T, \\
2 \\
K\left(\tau, \sigma_{1}\right) \text { für } \tau_{1}=\sigma_{1} \text { auf } S
\end{array}(\tau \text { in } T+S)\right.
$$

eingeführt. Setzt man

$$
\Psi(\tau)=\left\{\begin{array}{l}
\psi^{*}(\tau) \text { in } T, \\
\psi(\sigma) \text { auf } S,
\end{array} \quad F(\tau)=\left\{\begin{array}{l}
f^{*}(\tau) \text { in } T \\
f(\sigma) \text { auf } S
\end{array}\right.\right.
$$


und wie auf p. $218 d \tau=d \tau$ in $T,=d \sigma$ auf $S$, so lassen sich die Gleichungen $\left(40^{\prime}\right)$ und $\left(41^{\prime}\right)$ zu einer einzigen Gleichung

$$
\Psi\left(\tau^{\prime}\right)+\lambda \int_{T} K\left(\tau, \tau^{\prime}\right) \Psi(\tau) d \tau==F\left(\tau^{\prime}\right)
$$

zusammenziehen. Das Gleichungsystem $\left(40^{\prime}\right),\left(41^{\prime}\right)$ oder, was auf dasselbe hinausläuft, die Gleichung (44) ist als zu (4) adjungiert aufzufassen.

Die Lösung $\Psi\left(\tau^{\prime}\right)$ erweist sich, auch wenn $F\left(\tau^{\prime}\right)$ stetig ist, d. h. $f^{*}\left(\sigma^{\prime}\right)=f\left(\sigma^{\prime}\right)$ gilt, im Gegensatz zu $\varphi(\tau)$, der Lösung der Gleichung (4), als eine auf $S$ sprungweise unstetige Funktion, $\Psi^{* *}\left(\sigma^{\prime}\right) \neq \Psi\left(\sigma^{\prime}\right)$.

$$
\text { Ist freilich }
$$

so ist zugleich $\psi^{*}\left(\sigma^{\prime}\right)=\psi\left(\sigma^{\prime}\right)$, d. h. $\Psi(\tau)=\psi^{*}(\tau)$ für alle $\tau$ in $T+S$, und (44) geht über in die Gleichung

$$
\text { (46) } \psi\left(\tau^{\prime}\right)+\lambda \int_{T}^{1} K\left(\tau, \tau^{\prime}\right) \psi(\tau) d \tau+\lambda \int_{S}^{2} K\left(\sigma, \tau^{\prime}\right) \psi(\sigma) d \sigma=f\left(\tau^{\prime}\right) \text {, }
$$

die für alle $\tau^{\prime}$ in $T+S$ gilt. Ist auch noch $\stackrel{1}{K}\left(\tau, \tau^{\prime}\right)=\stackrel{1}{K}\left(\tau^{\prime}, x\right)$, so fällt (46) mit (4) zusammen. Die „belastete“ Integralgleichung (4) ist sich selbst adjungiert.

Es sei allgemeiner $\stackrel{2}{K}\left(\tau, \sigma^{\prime}\right)=p\left(\sigma^{\prime}\right) \stackrel{1}{K}\left(\tau, \sigma^{\prime}\right)$, und es möge $f\left(i^{\prime}\right)=p\left(\sigma^{\prime}\right) f^{*}\left(\sigma^{\prime}\right)$ sein. Man überzeugt sich leicht, daß jetzt $\psi\left(\sigma^{\prime}\right)=p\left(\sigma^{\prime}\right) \psi^{*}\left(\sigma^{\prime}\right)$ gilt.

Wir haben vorhin (vgl.p.221)

$$
f_{h}(\tau)=h f_{h}^{*}, \psi_{h}(\tau)=h \psi_{h}^{*} \text { in } T_{h}
$$

gesetzt und angenommen, daß $\psi_{h}^{*}\left(\tau^{\prime}\right)$ für $h \rightarrow 0$ für alle $\tau^{\prime}$ in jedem ganz in $T$ enthaltenen Bereiche gleichmäßig gegen eine in $T+S$ stetige Funktion $\psi^{*}\left(\tau^{\prime}\right)$ und zugleich $\psi_{h}\left(\tau^{\prime}\right)$ für alle $\tau^{\prime}$ in $T-T_{h}$ gegen eine auf $S$ stetige Funktion $\psi\left(\sigma^{\prime}\right)$ konvergiert.

Dies alles folgt leicht aus den Formeln (38) und (39), wenn man beachtet, daß nach (11) und (22)

$$
H_{h}\left(\tau, \tau^{\prime} ; \lambda\right)=\frac{\stackrel{1}{D_{h}}\left(\tau, \tau^{\prime} ; \lambda\right)}{D_{h}(\lambda)}+\frac{1}{h} \frac{\stackrel{2}{D_{h}}\left(\tau, \tau^{\prime} ; \lambda\right)}{D_{h}(\lambda)}
$$

gesetzt werden kann, und für $h \rightarrow 0$ gleichmäßig gilt

$$
\frac{\stackrel{1}{D}\left(\tau, \tau^{\prime} ; \lambda\right)}{D_{h}(\lambda)} \rightarrow \frac{\stackrel{1}{D}\left(\tau, \tau^{\prime} ; \lambda\right)}{D} \frac{\stackrel{D}{D}_{h}^{2}\left(\tau, \sigma^{\prime} ; \lambda\right)}{D_{h}(\lambda)} \rightarrow \frac{\stackrel{2}{D}\left(\tau, \sigma^{\prime} ; \lambda\right)}{D(\lambda)} .
$$

Wir finden (für alle $\tau^{\prime}$ in $T$ )

(49) $\psi^{*}\left(\tau^{\prime}\right)=f^{*}\left(\tau^{\prime}\right)-\lambda \int_{\tau} \frac{\stackrel{1}{D}\left(\tau, \tau^{\prime} ; \hat{\lambda}\right)}{D(\lambda)} f^{*}(\tau) d \tau-\lambda \int_{S} \frac{D^{1}\left(\sigma, \tau^{\prime} ; \lambda\right)}{D(\lambda)} f(\sigma) d \sigma$ sowie

(50) $\quad \psi(\sigma)=f\left(\sigma^{\prime}\right)-\lambda \int_{T} \frac{D^{2}\left(\tau, \sigma^{\prime} ; \lambda\right)}{D} \frac{i}{(\lambda)} f^{*}(\tau) d \tau-\lambda \int_{S} \frac{{\stackrel{D}{D}\left(\sigma, \sigma^{\prime} ; \hat{\lambda}\right)}^{\prime}}{D(\lambda)} f(\sigma) d \sigma$ und nach (33) und (43) kürzer

$$
\Psi\left(\tau^{\prime}\right)=F\left(\tau^{\prime}\right)-\lambda \int_{T+S} H\left(\tau, \tau^{\prime} ; \lambda\right) F(\tau) d \tau .
$$

Es ist leicht zu sehen, daß die Integralgleichungen (36) und (44) nur je eine Lösung haben (Unitätssatz). Hätte etwa (44) eine von (50) verschiedene Lösung, so gäbe es eine (auf $S$ im allgemeinen sprungweise unstetige) Lösung $\Psi_{0}\left(t^{\prime}\right)$ der homogenen Integralgleichung

$$
\Psi_{0}\left(\tau^{\prime}\right)+\lambda \int_{T+S} K\left(\tau, \tau^{\prime}\right) \Psi_{0}(\tau) d \tau=0 .
$$

Aus (36) und (52) folgt aber entsprechend durch Multiplikation mit $\Psi_{0}(\tau) \boldsymbol{d} \tau$ und $\Phi\left(\tau^{\prime}\right) \boldsymbol{d} \tau^{\prime}$ und Integration in naheliegender Weise

$$
\int_{T+S} F(\tau) \Psi_{0}(\tau) d \tau=0
$$

Nur, wenn diese Integralbeziehung erfüllt ist, würde (36) eine Lösung haben können, was einen Widerspruch darstellt.

Die im vorstehenden gewonnenen Formeln (vgl. namentlich die Beziehungen (36), (35'), (44), (51), (32), (29'), (31)) haben im Einklang mit der Bemerkung von Herrn E. Schmid und von A. KNESER dieselbe Form wie die klassischen Formeln der FreDHOLMschen Theorie. Sie könnten natürlich durch sinngemäße Übertragung der bekannten Schlüsse auch ohne Benutzung des Kernes $K_{h}\left(\tau, \tau^{\prime}\right)$ abgeleitet werden. Es ist einleuchtend, daß sich alle weiteren Ergebnisse sowohl der FredHolmschen als auch der SCHMidT- 
schen Theorie für die betrachteten „belasteten“ Integralgleichungen sinngemäß übertragen lassen.

Ist insbesondere im Gegensatz zu unseren bisherigen Annahmen $D(\lambda)=0$, so haben die homogenen Integralgleichungen

und

$$
\Phi(\tau)+\lambda \int_{T+S} K\left(\tau, \tau^{\prime}\right) \Phi\left(\tau^{\prime}\right) d \tau^{\prime}=0
$$

$$
\Psi\left(\tau^{\prime}\right)+\lambda \int_{T+S} K\left(\tau, \tau^{\prime}\right) \Psi(z) d \tau=0
$$

nicht identisch verschwindende Lösungen. Augenscheinlich sind die Lösungen der Gleichung (54) in $T+S$ stetig, diejenigen der Gleichung (55) ändern sich, wenn $\tau^{\prime}$ gegen $S$ konvergiert, im allgemeinen sprungweise.

Es seien $\tau^{(1)}, \ldots, \tau^{(p)}$ gewisse in $T+S$ gelegene Punkte, und es mögen $\stackrel{3}{K}_{1}\left(\tau, \tau^{(1)}\right), \ldots, \stackrel{3}{K}_{m}\left(\tau, \tau^{(p)}\right)$ in $T+S$ erklärte stetige Funktionen bezeichnen. Die Integralgleichung

$$
\begin{gathered}
\varphi(\tau)+\lambda \int_{T}^{1} K\left(\tau, \tau^{\prime}\right) \varphi\left(\tau^{\prime}\right) d \tau^{\prime}+\lambda \int_{S}^{2} K\left(\tau, \sigma^{\prime}\right) \varphi\left(\sigma^{\prime}\right) d \sigma^{\prime} \\
\quad+\lambda \sum_{l=1}^{p} K^{3}\left(\tau, \tau^{(l)}\right) \varphi\left(\tau^{(l)}\right)=f(\tau)
\end{gathered}
$$

$$
\Psi\left(\tau^{\prime}\right)+\lambda \int_{T+S} K\left(\tau, \tau^{\prime}\right) \Psi(\tau) d \tau=F(\tau)
$$

ist, auch wenn sich $F(\tau)$ durchaus stetig verhält, im allgemeinen auf $S$ sowie in den Punkten $\tau^{(l)}(l=1, \ldots, m)$ sprungweise unstetig. Das gleiche ist für $D(\lambda)=0$ bezüglich der Nullösungen $f$ der homogenen Integralgleichung

$$
\Psi\left(\tau^{\prime}\right)+\lambda \int_{T+S} K\left(\tau, \tau^{\prime}\right) \Psi(\tau) d \tau=0
$$

zu sagen.

Die vorstehenden Ergebnisse gelten sinngemäß, wenn das Linienintegral in (4) sich allgemeiner über eine endliche Anzahl stetig gekrümmten Kurvenbögen ${ }^{9}$ ) erstreckt. Eine Übertragung auf Räume von drei oder mehr Dimensionen ist naheliegend.

$\left.{ }^{9}\right)$ Allgemeiner: Kurvenbögen mit stetiger Tangente oder rektifizierbarer Jordanscher Kurvenstücke.

(Reçu par la Rédaction le 7. 9. 1931).

läßt sich ganz ähnlich wie die Gleichung (36) behandeln. Wir setzen $\Phi(\tau)=\varphi(\tau), F(\tau)=f(\tau)$ und für alle $\tau$ in $T+S$, sofern $\tau^{\prime}$ von $\tau^{(l)}(l=1, \ldots, p)$ verschieden ist

(57) $K\left(\tau, \tau^{\prime}\right)=\left\{\begin{array}{l}\stackrel{1}{K}\left(\tau, \tau^{\prime}\right) \text { für } \tau^{\prime} \text { in } T, \\ 2\left(\tau, \sigma^{\prime}\right) \text { für } \tau^{\prime}=\sigma^{\prime},\end{array} \quad \boldsymbol{d} \tau^{\prime}=\left\{\begin{array}{l}d \tau^{\prime} \text { in } T, \\ d \sigma^{\prime} \text { auf } S,\end{array}\right.\right.$

für $\tau^{\prime}=\tau^{(l)}(l=1, \ldots, p)$ aber

$$
K\left(\tau, \tau^{\prime}\right)=\stackrel{3}{K}\left(\tau, \tau^{\prime}\right), \quad \boldsymbol{d} \tau^{\prime}=1
$$

und erhalten wieder

$$
\Phi(\tau)+\lambda \int_{\tau+S} K\left(\tau, \tau^{\prime}\right) \Phi\left(\tau^{\prime}\right) d \tau^{\prime}=F(\tau) .
$$

Es möge $D(\lambda)$ von Null verschieden sein. Die Lösung der adjungierten Integralgleichung 